\title{
Effects of Microinjected Carbachol on the Antinociceptive Response to Noxious Heat Stimuli
}

\author{
Kenji Abe, Jun Kikuta, Masatoshi Kato, Kota Ishida, Toshiro Shigenaga, Kyoji Taguchi,* and \\ Tadashi MiYATAKE \\ Department of Neuroscience, Showa Pharmaceutical University; 3-3165 Higashitamagawagakuen, Machida, Tokyo \\ 194-8543, Japan. Received July 25, 2002; accepted October 25, 2002
}

\begin{abstract}
Injecting muscarinic receptor agonists into a specific area of the brainstem produces an antinociceptive response. The present study investigates whether direct injections of the cholinergic agonist, carbachol, into the rat nucleus reticularis gigantocellularis (NRGC)/nucleus reticularis gigantocellularis alpha (NRGC $\alpha$ ) of the rostral ventrolateral medulla evokes antinociception, and then examines the interference action of cholinergic antagonists in rats. Microinjections of carbachol $(0.75,1.5,3 \mu \mathrm{g} / \mathrm{site})$ prolonged hot plate (HP) and tail flick (TF) responses to noxious heat stimuli in a dose-dependent manner. The level of carbachol-induced antinociception during the HP and TF tests reached a maximum at 5-15 min after carbachol administration in all groups. Thereafter, the peak level progressively decreased and reached the baseline by the end of the experiment. Antinociception induced by carbachol at $3 \mu \mathrm{g} / \mathrm{site}$ was attenuated by the prior administration of the muscarinic receptor antagonist, atropine $(200,500 \mathrm{ng} / \mathrm{site})$. On the other hand, the nicotinic autonomic ganglion blocker, mecamylamine $(1,3 \mu \mathrm{g} / \mathrm{site})$, did not affect subsequent carbachol-induced antinociception. These results suggest that the antinociceptive effects induced by a microinjection of carbachol depend on muscarinic, but not nicotinic, mechanisms within the rat NRGC/NRGC $\alpha$.
\end{abstract}

Key words carbachol; microinjection; antinociception; nucleus reticularis gigantocellularis (NRGC); nucleus reticularis gigantocellularis alpha $(\mathrm{NRGC} \alpha)$; rat

The rostral ventrolateral medulla (RVLM), which includes the nucleus reticularis gigantocellularis (NRGC)/nucleus reticularis gigantocellularis alpha $(\mathrm{NRGC} \alpha)$ and the lateral reticular nucleus, plays a major role in maintaining tonic vasomotor tone and is critical in the reflex control of blood pressure. ${ }^{1,2}$ In addition, the RVLM modulates one of the physiological functions of antinociception via cholinergic neurons. ${ }^{3,4)}$ Immunohistochemical studies have shown that the RVLM is extensively innervated with cholinergic neurons ${ }^{5)}$ that mainly project from the pedunculopontine tegmental nucleus (PPT), ${ }^{6}$ then efferently extend to the spinal cord. In fact, small-medium cholinergic neurons and choline acetyltransferase mRNA have been identified in small cells of the NRGC/nucleus reticularis paragigantocellularis (NRPG). ${ }^{7)}$ These observations suggest that cholinergic neurons play an important role in the RVLM, including NRGC/NRGC $\alpha$.

Anticholinesterase and cholinergic mimetics exert analgesic effects in humans ${ }^{8,9)}$ monkeys, ${ }^{10)}$ rats and mice. ${ }^{11)}$ Moreover, the administration of carbachol into the nucleus raphe magnus induces pronounced antinociception that is reversed by atropine and muscarinic antagonists. ${ }^{12)}$ Injections of muscarinic agonists into the spinal cord also have antinociceptive effects on responses to noxious heat stimuli via muscarinic receptors. ${ }^{11,13)}$ We reported that either systemically-administered or RVLM-injected morphine enhances ACh release in the RVLM, and that the local application of morphine via a microdialysis probe induces an increase in both tail withdrawal and hot-plate responses. ${ }^{14)}$ Thus, the cholinergic system in the central nervous system may be considered to have a key role in endogenous pain control.

Thus, the present study investigates whether a direct injection of the cholinergic agonist, carbachol, into the NRGC/NRGC $\alpha$ evokes antinociception, and examines the participation of antinociceptive events in rats.

\section{MATERIALS AND METHODS}

Animals Male Wistar rats, weighing 250 to $350 \mathrm{~g}$ at the time of the experiments, were purchased from Charles River Japan, Inc. All animals were housed individually under automatically controlled environmental conditions and $12 \mathrm{~h}$ light-dark cycles, and were handled in accordance with the guidelines for animal care and use published by the National Institute of Health. The rats had free access to food and water. All animals were quarantined in centralized animal facilities for at least seven days after arrival from the supplier.

Preparation for Microinjection After an acclimation period, the animals were anesthetized with sodium pentobarbital $(50 \mathrm{mg} / \mathrm{kg}$, i.p.) and placed in a stereotaxic apparatus. A midline incision was made on the scalp, and a burr hole was drilled for a 27-gauge stainless-steel cannula (guide cannula) that was firmly placed using dental acrylic and anchored to the skull using stainless-steel screws. Drugs were injected through a 30-gauge stainless-steel cannula, the tip of which extended $4 \mathrm{~mm}$ beyond the tip of the guide cannula into the NRGC/NRGC $\alpha$ according to the atlas of Paxinos and Watson. ${ }^{15)}$ The coordinates of the NRGC/NRGC $\alpha$ were: bregma $-11.5 \mathrm{~mm}$, lateral $0.9 \mathrm{~mm}$, and depth $-10.5 \mathrm{~mm}$. To confirm that no abnormalities resulted from the procedure, the animals were observed for one week after surgery and prior to drug administration.

Drug Administration The following drugs were microinjected: the non-selective cholinergic agonist, carbamylcholine chloride (carbachol, Sigma); the specific muscarinic antagonist, atropine sulfate (Tokyokasei) and the nicotinic autonomic ganglion blocker, mecamylamine hydrochloride (Sigma). All drugs were dissolved immediately before use in sterile physiological saline and filtered through a $0.2 \mu \mathrm{m}$ pore size membrane. The injection cannula was connected to a Hamilton microsyringe via PE-10 polyethylene tubing and 
filled with either saline or drug solution. A $0.5 \mu 1$ volume of the solutions was injected over $60 \mathrm{~s}$ into the NRGC/NRGC $\alpha$, then the cannula was carefully withdrawn.

Carbachol-Induced Antinociception and Effects of Cholinergic Antagonists The hot plate (HP) and tail flick (TF) tests assessed the nociceptive response. For the HP test, rats were placed onto a heated plate set at $55^{\circ} \mathrm{C}$ and the latency of paw licking or jumping was measured. A cut-off time of $30 \mathrm{~s}$ was established to avoid tissue injury. The animals were held in the polycarbonate apparatus for the TF test with the tail protruding. The tail was blackened with ink on the ventral surface at about $3 \mathrm{~cm}$ from the tip, and three spots were marked at intervals of $1 \mathrm{~cm}$. High-intensity light was randomly applied to the blackened spots. The time interval between the onset of heating and the tail withdrawal response was electronically measured. A cut-off time of $10 \mathrm{~s}$ was established to minimize the probability of tissue damage. The average HP and TF response latency values from three consecutive trials before drug administration were used as the baseline.

For the dose-response study, $5 \mathrm{~min}$ after the baseline response latencies were obtained (defined as $t=-15$ ), saline was injected into the NRGC/NRGC $\alpha$, and test response latencies were recorded every $5 \mathrm{~min}$. Immediately after the third test (defined as $t=0$ ), the NRGC/NRGC $\alpha$ was administered with saline or carbachol (at doses of $0.75,1.5$ or $3 \mu \mathrm{g} / \mathrm{site}$ ) through an injection cannula. Test latencies were determined from +5 min later, then at 5-min intervals up to $+20 \mathrm{~min}$. Thereafter, latency was measured at 10-min intervals up to $+60 \mathrm{~min}$. The effects of atropine and mecamylamine were investigated after antinociception was induced with $3 \mu \mathrm{g} /$ site of carbachol. These drugs were microinjected $15 \mathrm{~min}$ before carbachol.

Location of Injection Site After all experiments, the animals were deeply anesthetized with sodium pentobarbital. Correct placement of the microinjections was verified in each animal by injecting methylene blue into the NRGC/NRGC $\alpha$. Animals were then sacrificed with an overdose of sodium pentobarbital. The brains removed from each rat were fixed overnight in $10 \%$ formalin, and cut into cryostat sections.

Data Analysis The HP and TF responses of each rat were converted to percent maximum possible effect (\%MPE) using the formula:

$$
\% \mathrm{MPE}=\frac{\text { post }- \text { drug latency }- \text { pre-drug latency }}{\text { cut-off time }- \text { pre-drug latency }} \times 100
$$

Data are expressed as means \pm standard error (S.E.) of the $\%$ MPE for each group. To determine the effect of cholinergic antagonists on carbachol-induced antinociception, the significance of difference between the groups was analyzed by repeated measurement two-way analysis of variance (ANOVA), followed by Dunnett's multiple comparison tests. A value of $p<0.05$ was regarded as statistically significant.

\section{RESULTS}

Effect of Microinjected Carbachol on Antinociception The administration of carbachol into the NRGC/NRGC $\alpha$ induced a dose-dependent antinociceptive response (Fig. 1). Levels of \%MPE during the HP $(20.4 \pm 10.6,43.5 \pm 9.8 \%)$ and $\mathrm{TF}(39.7 \pm 15.4,84.2 \pm 12.8 \%)$ tests reached a maximum
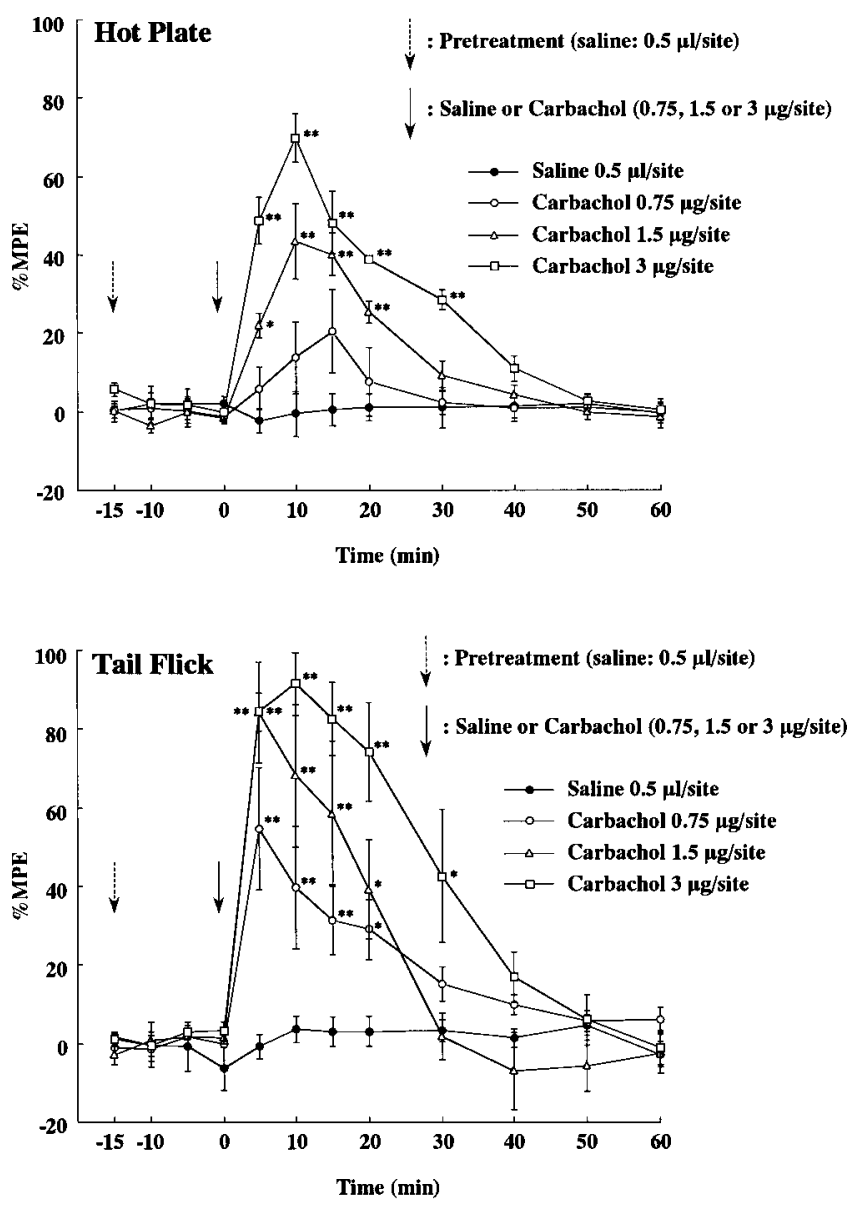

Fig. 1. Time Course of Antinociception Produced by Microinjecting Carbachol into the NRGC/NRGC $\alpha$

Carbachol was injected at the times indicated by arrows. Saline $(0.5 \mu \mathrm{l})$ was microinjected into NRGC/NRGC $\alpha$ followed by carbachol $(0.75,1.5,3 \mu \mathrm{g} / 0.5 \mu \mathrm{l} ; n=5, n=5$, $n=6)$ or saline $(0.5 \mu \mathrm{l} ; n=5)$ at the same site $15 \mathrm{~min}$ later. Asterisks indicate significant differences $(* p<0.05, * * p<0.01)$ between the saline and carbachol. Each point represents the mean \pm S.E.M.

$5-15 \mathrm{~min}$ after the administration of 0.75 and $1.5 \mu \mathrm{g} /$ site of carbachol. Thereafter, the peak progressively decreased and reached a baseline value at $+30-+40 \mathrm{~min}$ in both tests. Carbachol ( $3 \mu \mathrm{g} / \mathrm{site})$ induced more potent and long-lasting antinociceptive effects in both the HP and TF responses. Maximal levels of \%MPE in the HP and TF test were $69.9 \pm 6.2 \%$ and $91.5 \pm 7.9 \%$, respectively, +10 min after carbachol administration. Antinociceptive effects of carbachol in the HP and TF test decreased with elapsed time and returned to the baseline value, although the interval was longer in the group given $1.5 \mu \mathrm{g} /$ site (baseline values in the HP and $\mathrm{TF}$ tests were reached $+50 \mathrm{~min}$ after carbachol administration).

Effect of Cholinergic Antagonists on Carbachol-Induced Antinociception Atropine (200 ng/site) moderately inhibited the subsequent carbachol-induced antinociceptive response. Maximal levels of \%MPE during the HP and TF tests in this group were $46.7 \pm 7.2 \%$ and $62.1 \pm 13.5 \%$, respectively (Fig. 2). Atropine $(500 \mathrm{ng} /$ site) more potently inhibited carbachol-induced antinociception, and the values of $\% \mathrm{MPE}$ in the HP $(25.6 \pm 3.8 \%)$ and TF $(26.8 \pm 5.6 \%)$ responses were clearly diminished. On the other hand, 1 and $3 \mu \mathrm{g} / \mathrm{site}$ of mecamylamine, a nicotinic receptor antagonist, did not influence carbachol-induced antinociception (Fig. 3). 

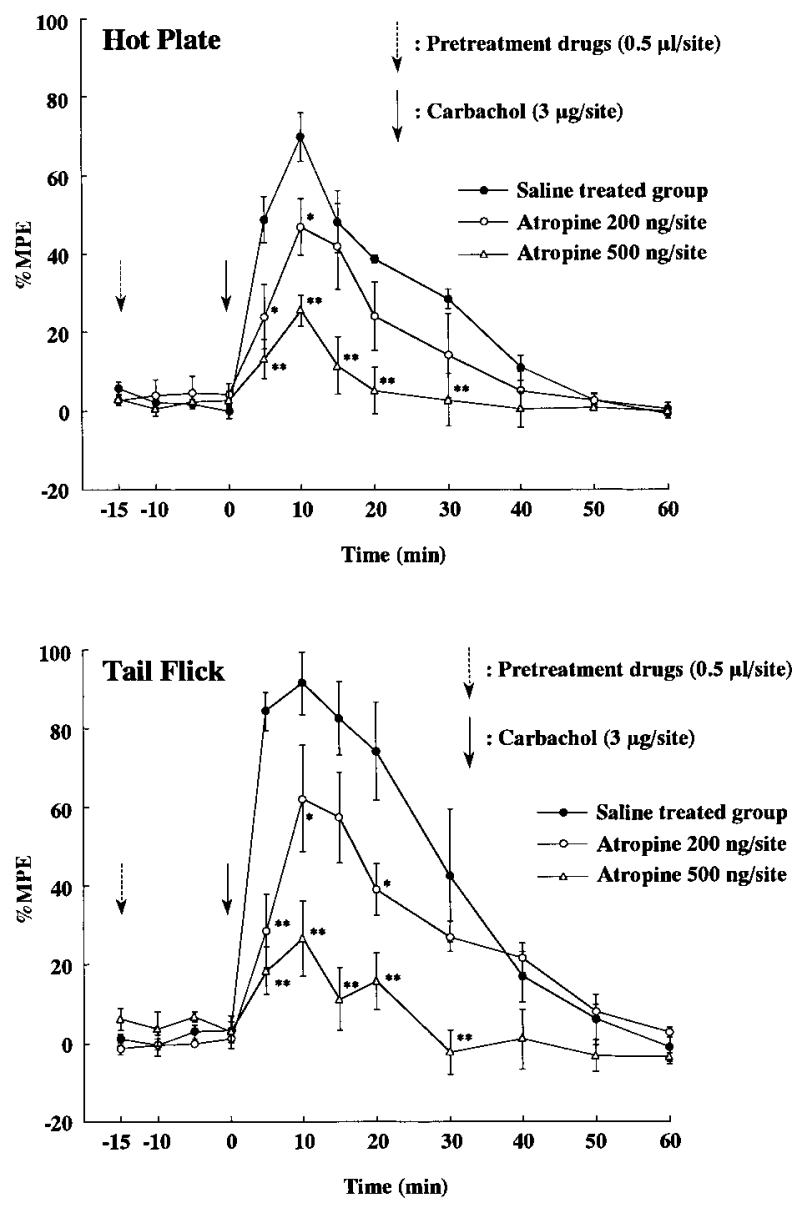

Fig. 2. Effects of Atropine on Subsequent Antinociception Induced by Carbachol

Atropine (either 200 or $500 \mathrm{ng} / 0.5 \mu \mathrm{l} ; n=6$ ) was microinjected $15 \mathrm{~min}$ before carbachol $(3 \mu \mathrm{g} / 0.5 \mu \mathrm{l})$ at the times indicated by arrows. Atropine antagonized carbachol-induced antinociception. Asterisks indicate significant differences $(* p<0.05, * * p<0.01)$ between the presence and absence of prior atropine. Each point represents the mean \pm S.E.M.
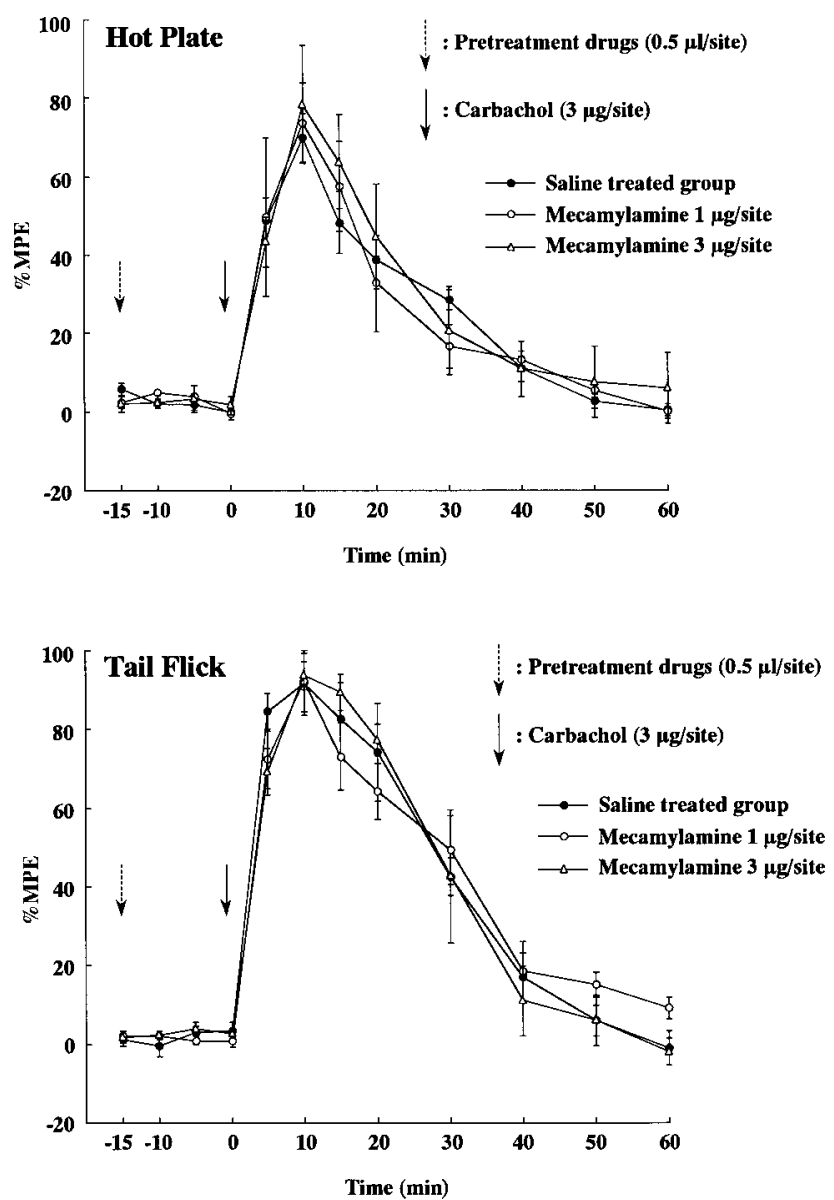

Fig. 3. Effects of Mecamylamine on Subsequent Antinociception Induced by Carbachol

Mecamylamine ( 1 or $3 \mu \mathrm{g} / 0.5 \mu \mathrm{l} ; n=5$ ) was microinjected $15 \mathrm{~min}$ before carbachol $(3 \mu \mathrm{g} / 0.5 \mu \mathrm{l})$ at the same site. Mecamylamine did not alter carbachol-induced antinociception. Each point represents the mean \pm S.E.M.

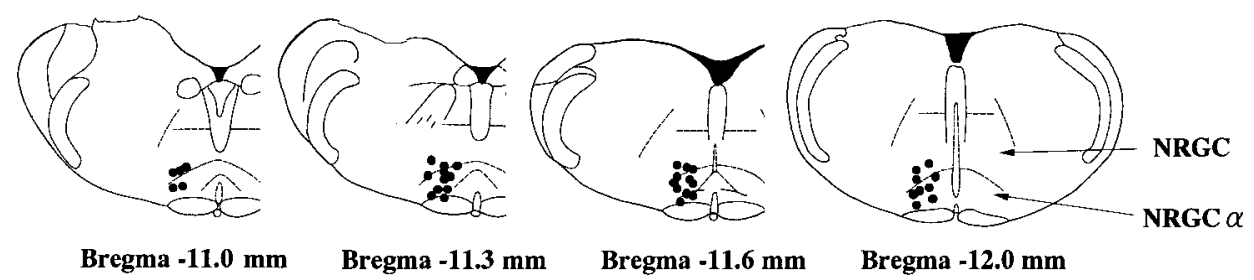

Fig. 4. Location of the NRGC/NRGC $\alpha$ Microinjection Site

All injection sites were located precisely within NRGC/NRGC $\alpha$ regions.

Location of the Injection Site Figure 4 shows histological verification of the microinjection site. We confirmed that all microinjection sites were within the region of the NRGC/NRGC $\alpha$.

\section{DISCUSSION}

The NRGC/NRGC $\alpha$ is involved in the RVLM, and this area processes not only cardiovascular modulation but also nociceptive information via noradrenergic or serotonergic pathways. ${ }^{16-18)}$ Cholinergic neurons in the NRGC/NRGC $\alpha$ mainly project from the $\mathrm{PPT},{ }^{6}$ and microinjections of carbachol into the PPT also produce dose-dependent antinocicep- tion. ${ }^{12,19,20)}$ In the present study, the results of the HP and TF tests showed that carbachol microinjected into the NRGC/NRGC $\alpha$ induced antinociception in a dose-related manner. Our results indicated that cholinergic neurons in the NRGC/NRGC $\alpha$ participate in antinociceptive responses to noxious heat stimuli. Electrophysiological studies have shown that electrical stimulation of the NRGC/NRGC $\alpha$ inhibits the spinal nociceptive reflex and the spinal dorsal horn neuron response to peripheral stimulation. ${ }^{21,22)}$ Some evidence shows that cholinergic neurons of the NRGC/NRGC $\alpha$ participate in the regulation of antinociceptive information. Thus, systemic or intrathecal administration of cholinomimetic drugs, particularly anticholinesterase, enhances 
the antinociception caused by opioids administered to humans and other animals. ${ }^{23,24)}$ Moreover, the injection of muscarinic receptor agonists into a specific part of the brainstem, such as the dorsal periaqueductal gray, or nucleus raphe magnus, produces an antinociceptive response that is antagonized by muscarinic or nicotinic antagonists ${ }^{19,25,26)}$ or by an intrathecal injection of cholinergic antagonists. ${ }^{11)}$ We demonstrated that the local in vivo microdialysis application of morphine into the RVLM, which includes the NRGC/ NRGC $\alpha$, increases antinociceptive responses, and enhances ACh release. ${ }^{14)}$ Thus, the present study examined the direct cholinergic stimulation of NRGC/NRGC $\alpha$-induced antinociceptive behavior, in which the muscarinic and nicotinic receptors participated more potently in cholinergic stimulatedantinociception.

Cholinergic antinociception results from the activation of muscarinic or nicotinic mechanisms and depends on a site in the central nervous system that is activated by cholinergic agonists. We found that the increased HP and TF responses produced by microinjecting carbachol into the NRGC/ NRGC $\alpha$ were blocked by microinjecting the muscarinic antagonist atropine, but not by the nicotinic receptor antagonist, mecamylamine. Similarly, a microinjection of carbachol into the nucleus raphe magnus produces dose-dependent antinociception that is reversed by atropine. ${ }^{12,19,20)}$ In contrast, Guimaraes et al. have shown that a microinjection of carbachol into the dorsal periaqueductal gray increases the TF latency of rats, and that carbachol-induced antinociception is inhibited by mecamylamine, but not by atropine. ${ }^{26)}$ The nicotinic receptor antagonist, mecamylamine did not affect carbachol-induced antinociception in our study. In addition, nicotine-induced antinociception depends upon intact acetylcholine release and upon receptor interactions at nicotinic and at muscarinic receptor sites in the nucleus raphe magnus. ${ }^{27)}$ Thus, the effects of carbachol from the brainstem, including NRGC/NRGC $\alpha$, may depend on the activation of a different cholinergic receptor. Our data suggest that the antinociceptive effects of carbachol microinjected into the NRGC/NRGC $\alpha$ are mediated by the muscarinic receptor to noxious heat stimuli. Further study is required to determine the contribution of cholinergic neurons to nociception and their relationship to other neurons.

In conclusion, carbachol microinjected into the NRGC/
NRGC $\alpha$ induces antinociceptive effects via cholinergic muscarinic receptors. These results suggest that cholinergic neurons in the NRGC/NRGC $\alpha$ play an important role in regulating antinociception.

\section{REFERENCES}

1) Ross C. A., Armstrong D. M., Ruggiero D. A., Pickel V. M., Joh T. H., Reis D. J., Neurosci. Lett., 25, 257-262 (1981).

2) Kubo T., Taguchi K., Sawai N., Ozaki S., Hagiwara Y., Brain Res. Bull., 42, 199-204 (1997).

3) Ossipov M. H., Gebhart G. F., Brain Res., 384, 282-293 (1986).

4) Zhuo M., Gebhart G. F., J. Neurophysiol., 67, 1599-1614 (1992).

5) Giuliano R., Ruggiero D. A., Morrison S., Ernsberger P., Reis D. J., J. Neurosci., 9, 923-942 (1989).

6) Yasui Y., Cechetto D. F., Saper C. B., Brain Res., 517, 19-24 (1990).

7) Lauterborn J. C., Isackson P. J., Montalvo R., Gall C. M., Mol. Brain Res., 17, 59-69 (1993).

8) Christensen E. M., Gross E. G., J. Am. Med. Ass., 137, 594-598 (1948).

9) Hood D. D., Eisenach J. C., Tuttle R., Anesthesiology, 82, 331-343 (1995).

10) Pert A., Psychopharmacologica (Berlin), 44, 131-137 (1975).

11) Iwamoto E. T., Marion L., J. Pharmacol. Exp. Ther, 266, 329-338 (1993).

12) Brodie M. S., Proudfit H. K., Brain Res., 291, 337-342 (1984).

13) Naguib M., Yaksh T. L., Anesth. Analg., 85, 847-853 (1997).

14) Taguchi K., Kato M., Kikuta J., Abe K., Chikuma T., Utsunomiya I., Miyatake T., J. Pharmacol. Exp. Ther., 289, 1539-1544 (1999).

15) Paxinos G., Watson C., "The Rat Brain in Stereotaxic Coordinates," 2nd ed., Academic Press, New York, 1986.

16) Kuo T. B., Yang C. C., Chan J. Y., Tsai H. F., Chan S. H., J. Biomed. Sci., 3, 338-347 (1996).

17) Yang C. H., Shyr M. H., Tan P. P., Chan S. H., J. Pharmacol. Exp. Ther, 279, 795-802 (1996).

18) Kiefel J. M., Cooper M. L., Bodner R. J., Brain Res., 597, 331-338 (1992).

19) Brodie M. S., Proudfit H. K., Brain Res., 371, 70-79 (1986).

20) Klamt J. G., Prado W. A., Brain Res., 549, 9-18 (1991).

21) Sandkuhler J., Gebhart G. F., Brain Res., 305, 67-76 (1984).

22) Zhuo M., Gebhart G. F., J. Neurophysiol., 78, 746-758 (1997).

23) Zhuo M., Gebhart G. F., Brain Res., 535, 67-78 (1990).

24) Hood D. D., Mallak K. A., James R. L., Tuttle R., Eisenach J. C., J. Pharmacol. Exp. Ther, 282, 86-92 (1997).

25) Iwamoto E. T., Marion L., J. Pharmacol. Exp. Ther., 269, 699-708 (1994).

26) Guimaraes A. P., Guimaraes F. S., Prado W. A., Brain Res. Bull., 51, $471-478$ (2000).

27) Iwamoto E. T., J. Pharmacol. Exp. Ther., 257, 120-133 (1990). 